\title{
Slice-direction geometric distortion evaluation and correction with reversed slice-select gradient acquisitions
}

Anna I. Blazejewska ${ }^{1,2,{ }^{*}}$, Thomas Witzel ${ }^{3}$, Jesper L.R. Andersson ${ }^{4}$, Lawrence L. Wald ${ }^{1,2,5}$, and Jonathan R.

$$
\text { Polimeni }{ }^{1,2,5}
$$

1: Athinoula A. Martinos Center for Biomedical Imaging, Massachusetts General Hospital, Charlestown,

$$
\text { Boston, MA, USA }
$$

2: Department of Radiology, Harvard Medical School, Boston, MA, USA

$$
\text { 3: Q Bio, Inc., Redwood City, CA, USA }
$$

4: Wellcome Centre for Integrative Neuroimaging, FMRIB, Nuffield Department of Clinical Neurosciences, University of Oxford, Oxford, UK

5: Harvard-MIT Division of Health Sciences and Technology, Massachusetts Institute of Technology, Cambridge, MA, United States

*Correspondence should be directed to:

Anna Blazejewska, Ph.D.

MGH Athinoula A. Martinos Center for Biomedical Imaging

Bldg $14913^{\text {th }}$ St Rm 2301

Charlestown MA, 02129 USA

E-mail address: ABLAZEJEWSKA@mgh.harvard.edu 


\section{Abstract}

2 Accurate spatial alignment of MRI data acquired across multiple contrasts in the same subject is often

3 crucial for data analysis and interpretation, but can be challenging in the presence of geometric distortions

4 that differ between acquisitions. It is well known that single-shot echo-planar imaging (EPI) acquisitions

5 suffer from distortion in the phase-encoding direction due to $B_{0}$ field inhomogeneities arising from tissue

6 magnetic susceptibility differences and other sources, however there can be distortion in other encoding

7 directions as well in the presence of strong field homogeneities. High-resolution ultrahigh-field MRI

8 typically uses low bandwidth in the slice-encoding direction to acquire thin slices and, when combined

9 with the pronounced $B_{0}$ inhomogeneities, is prone to an additional geometric distortion in the slice

10 direction as well. Here we demonstrate a presence of this slice distortion in high-resolution 7T EPI

11 acquired with a novel pulse sequence allowing for the reversal of the slice-encoding gradient polarity that

12 enables the acquisition of pairs of images with equal magnitudes of distortion in the slice direction but

13 with opposing polarities. We also show that the slice-direction distortion can be corrected using gradient

14 reversal-based method applying the same software used for conventional corrections of phase-encoding

15 direction distortion. 


\section{Introduction}

Many neuroimaging studies using MRI frequently leverage multiple imaging protocols with varying complementary contrasts. This can enable the extraction of more functional or structural information at any given anatomical location than what can be achieved with any single modality alone, which is a key advantage of MRI over other noninvasive imaging technologies. To achieve this, accurate alignment of these MRI data across the different contrasts acquired is critical. Head motion often occurs between scans, and rigid registration can be employed to align the datasets-by utilizing various available methods that can account for the different image contrasts-however this alignment is far more challenging in the presence of differential geometric distortion between these acquisitions. In many cases, these geometric distortions also can change with head position, further exacerbating error in alignment.

The most prevalent source of geometric distortion in MRI is inhomogeneity of the main magnetic field, $B_{0}$, which for modern MRI scanners is predominantly induced by magnetic susceptibility differences within the volunteer's head mainly found in regions near to air-tissue interfaces (e.g. at the sinuses, ear canals, and oral cavity). These local magnetic field variations cause field gradients that interfere with the image encoding gradients and thereby result in errors in image encoding such as voxel shift errors, which manifest as nonrigid geometric distortion (i.e., local expansion or compression) of the resulting image. For a given local $B_{0}$ field offset, these image encoding errors scale with image encoding bandwidth, such that a lower encoding bandwidth results in a greater vulnerability to distortion and a larger voxel shift in the presence of field inhomogeneity.

Single-shot Echo Planar Imaging (EPI) is widely used for acquiring various MRI contrasts such as functional, diffusion and perfusion weighted imaging, and increasingly for anatomical imaging as well. EPI is well known to be vulnerable to these geometric distortions, which are primarily along the phaseencoding direction; in EPI, not only is this the encoding direction with the lowest bandwidth, but also in most applications of EPI the phase-encoding bandwidth is sufficiently low that distortions are clearly apparent, with maximum voxel displacements in the centimeter range. Phase encoding in EPI has relatively low encoding bandwidth (such as $30 \mathrm{~Hz} /$ pixel) compared to the relatively high frequency encoding bandwidths typically used in conventional (non-EPI) acquisitions (such as $600 \mathrm{~Hz} /$ pixel), thus geometric distortion is most often considered to be only in the phase-encoding direction in EPI, and for most non-EPI applications distortion in the frequency-encoding direction is negligible (Jezzard, 2012). While the distortion in the phase-encoding direction in EPI can be partly mitigated with parallel imaging acceleration (de Zwart et al., 2002; Griswold et al., 1999), which increases the effective phase-encoding bandwidth, the undersampling used in accelerated acquisitions brings with it a fixed penalty of decreased 
signal-to-noise ratio (SNR), and high acceleration factors can introduce severe artifacts even with modern receive coil arrays, therefore acceleration alone cannot fully remove the distortion in EPI. For this reason, EPI distortion must be addressed in post processing. Conventionally, post-hoc correction of the geometric distortion is performed based on an estimation of the voxel shifts induced by the $B_{0}$ magnetic field inhomogeneity during the image acquisition, where the field offsets can be calculated from a separately acquired B $B_{0}$ map (Jezzard and Balaban, 1995). Another approach is based on the "reversed gradient" method that, instead of using an explicit $B_{0}$ field map, infers the distortion using a pair of two EPI scans acquired with the phase-encoding gradients played out with opposite polarities, resulting in two images with equal magnitudes of distortion but in opposite directions, from which non-linear image registration can be used to simultaneously estimate and correct the distortion (Andersson et al., 2003; Bowtell et al., 1994; Chang and Fitzpatrick, 1992; Holland et al., 2010; Morgan et al., 2004).

While distortion in the phase-encoding and frequency-encoding directions have been thoroughly considered in previous work, the distortion in the slice-encoding direction for 2D slice-by-slice imaging has received some attention (Studholme et al., 2000), but is becoming more important as it may be increasingly present in modern MRI applications. For example, high-resolution (sub-millimeter) fMRI uses thin imaging slices which are usually acquired by maximizing slice-select gradient strength; however once the maximum strength is reached, a decrease of the slice-encoding gradient bandwidth is required to further narrow the range or excited frequencies, which could lead to the additional geometric distortions and "slice bending" in the slice direction. For conventional slice-selective pulses, slice thickness (TH) is determined by the slice-encoding gradient strength $\left(G_{z}\right)$ and slice-select RF pulse bandwidth (BW) such that $T H=B W / \gamma G_{z}$. While the value of $G_{z}$ is usually dictated by the desired slice thickness, the RF pulse bandwidth is related to the pulse duration $(D)$ and the time-bandwidth product (TBW), i.e., TBW $\equiv D \cdot B W$. In sub-millimeter imaging, because $G_{z}$ typically cannot be further increased, the slice thickness can be decreased by modifying the RF pulse design either by reducing TBW (which leads to degradation of the slice profile), or by increasing the pulse duration (which lowers SAR but increases the minimal echo time, TE). Thus, to decrease slice thickness, often one must resort to reducing the RF excitation pulse bandwidth. While stronger gradient coils help to achieve distortion-free thin slices, they bring a penalty of increased Peripheral Nerve Stimulation and potential eddy currents. High-resolution $\mathrm{fMRI}$ is commonly performed at ultra-high magnetic field strengths $\left(\begin{array}{ll}\geq \mathrm{T} & \mathrm{T}\end{array}\right)$ where susceptibility-induced $\mathrm{B}_{0}$ field inhomogeneities are stronger than at conventional field strengths, suggesting that this imaging modality may be particularly vulnerable to slice distortion. In addition, in many applications a low slice-select RF pulse bandwidth may also be desirable to decrease power deposition (SAR). Slice distortion due to field 
inhomogeneity has previously been reported in MRI studies conducted using conventional field strengths as well (such as $1.5 \mathrm{~T}$ ) (Sumanaweera et al., 1993) and in the presence of metal implants where magnetic susceptibility offsets are substantially higher than those between different tissue types (Hargreaves et al., 2011; Hopper et al., 2006; Lu et al., 2009).

To characterize this slice distortion and present a method for correcting it, here we use a novel acquisition that acquires pairs of images with reversed polarity of the slice-encoding gradient to create image pairs with equal slice distortions in opposing directions, and then demonstrate that typical 7T fMRI acquisitions do suffer from noticeable geometric distortion in the slice-encoding direction, which will result in alignment errors when registering these data to other datasets, e.g., to anatomical reference data typically acquired using 3D encoding. Our method is analogous to the reversed-gradient method used for removing distortion in the phase-encoding or frequency-encoding directions, and here it is applied in the slice-encoding direction. We demonstrate a correction of these slice distortions with our method, and evaluate it using both spin-echo (SE) or gradient-echo (GE) based data acquired with slice gradients and RF pulse (excitation and refocusing) bandwidths that are matched those used in the target fMRI protocol. Because we use, for correction, the popular topup method implemented in the FSL package (Andersson et al., 2003; Smith et al., 2004), we have named our method overeasy (Blazejewska et al., 2017). While we demonstrate our approach using high-resolution EPI data, it is applicable to any 2D multi-slice data suffering from geometric distortion in the slice direction, which becomes more pronounced at higher spatial resolutions and higher magnetic field strengths.

\section{Materials and methods}

Seven healthy volunteers (4F/3M, $26 \pm 7$ y.o.) participated in this study after providing written informed consent in accordance with our institution's Human Research Committee; the study protocol was approved by the Institutional Review Board of Massachusetts General Hospital.

All data were acquired on a whole-body 7T scanner (MAGNETOM, Siemens Healthcare, Erlangen, Germany) equipped with a "short-z FOV" body gradient coil (SC72) and an inhouse-built 32-channel coil array for receive and a single-channel birdcage coil for transmit (Keil et al., 2010). During each experimental session multiple EPI scans were acquired following standard automatic $B_{0}$ shimming. 


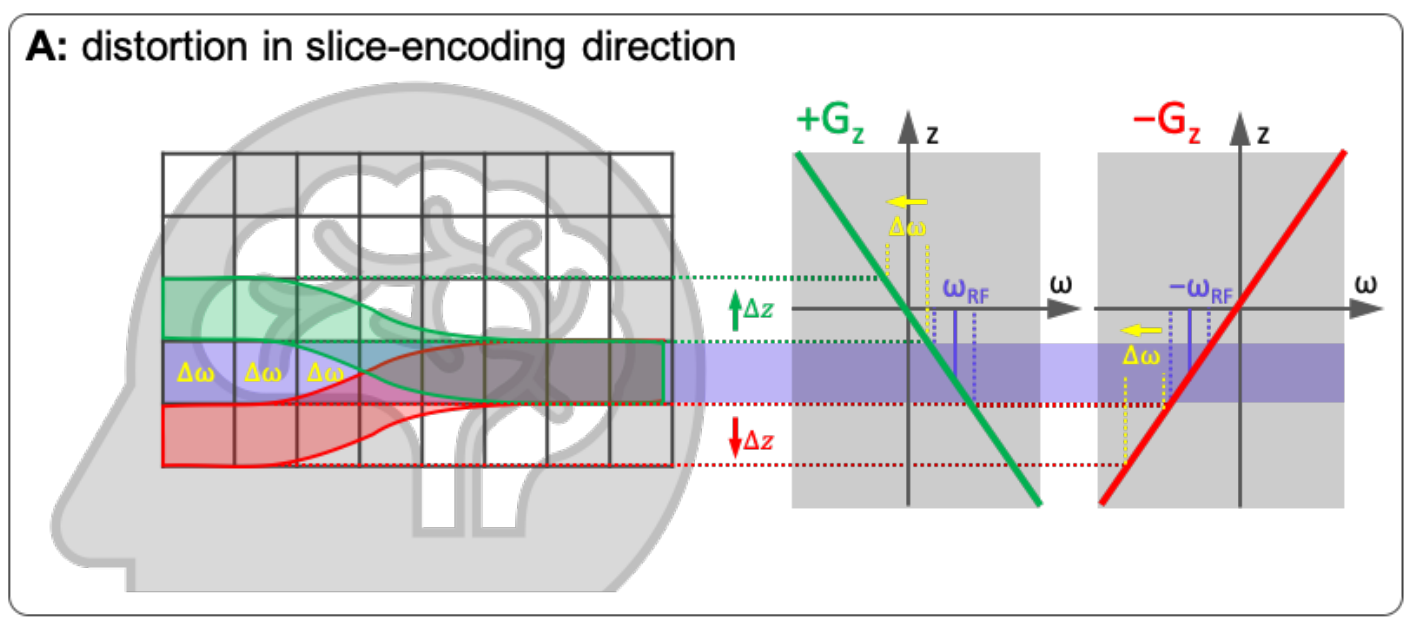

\section{B: single-slice displacement in SE EPI}

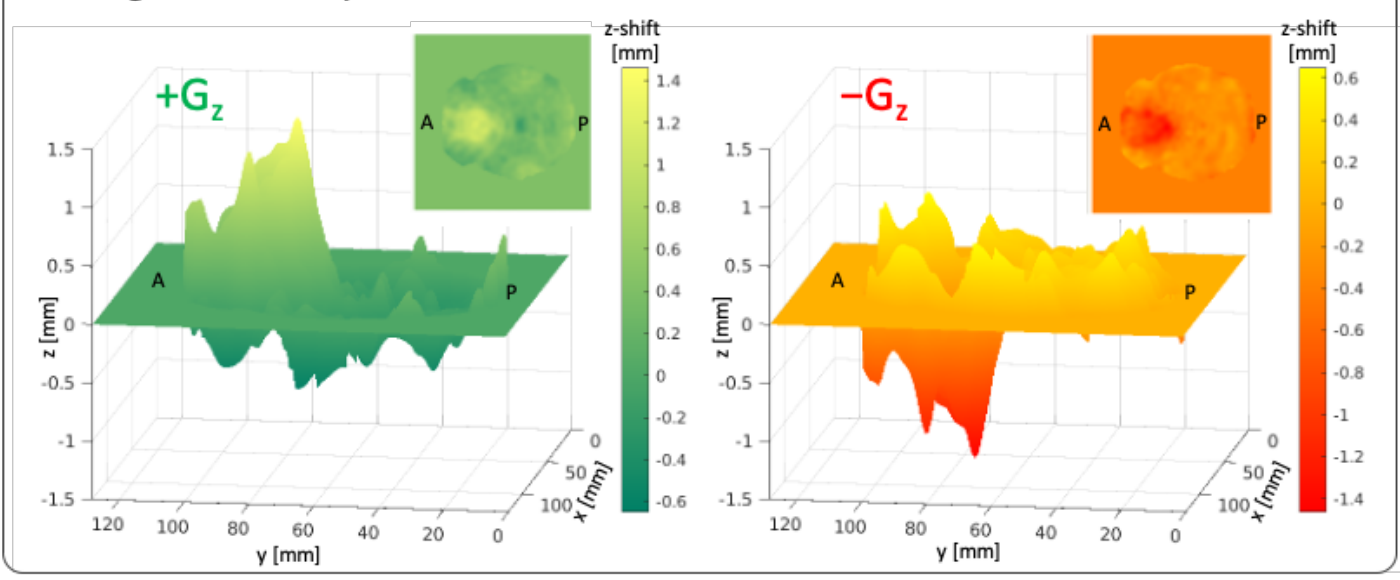

Figure 1. (A) Explanation of slice-encoding distortion due to local $B_{0}$ field offsets and the reverse-gradient method applied to the slice-select direction. The slice-select gradient establishes a mapping between frequency and position along the sliceselect direction (here, the $z$ direction). A given slice location is excited by an RF pulse at the corresponding frequency. In the absence of local $B_{0}$ field offsets, either a positive $z$ gradient $\left(+G_{z}\right)$ or a negative $z$ gradient $\left(-G_{z}\right)$ can be applied, and the desired slice location can be excited with an RF pulse using the appropriate center frequency, $+\omega_{\mathrm{RF}}$ or $-\omega_{\mathrm{RF}}$, respectively. In the presence of local $B_{0}$ field offsets, the location of the excited slice will be shifted, and the direction of the shift will depend on the polarity of the slice-selective gradient. In this case, the direction of the shift will depend on whether the $+G_{z}$ gradient or the $-G_{z}$ gradient is used, and the magnitude of the shift will depend on the local $B_{0}$ field offset $\Delta \omega$ as well as the gradient amplitude and the RF pulse bandwidth. A desired slice location without the shift is marked with purple, while actual slice locations with the shift occurring in presence of $+G_{z}$ and $-G_{z}$ are marked with green and red, respectively. (B) Example of distortion-induced displacement (z-shift) of the voxels in the slice-encoding direction within a single axial slice, caused by local $B_{0}$ field offsets, for images acquired with two opposite slice-encoding gradient polarities. In this example the shift is largest in the region at the front of the brain (near the frontal sinuses), and manifests as a positive z-shift when using the $+G_{z}$ gradient and a negative $z$-shift when using the $-G_{z}$ gradient. 
The overeasy method used in this study consists of acquiring pairs of EPI data with reversed sliceselect gradient polarity, with appropriate shifting of the RF pulse center frequency, which causes the voxel displacement direction to alternate between the two acquisitions, as illustrated in Figure 1. Note that the images were not acquired purely axially, and were tilted somewhat to be roughly aligned to the brain's AC-PC axis, therefore the slice-select gradient was not along a single gradient axis thus the gradient was a combination of the physical $G_{x}, G_{y}$ and $G_{z}$ gradients. Because the only parameter to change between this pair of images is the slice-select gradient polarity, the differential distortion between these images is in the direction perpendicular to the slice plane. Because these two images are acquired immediately after one another, we assume that the $B_{0}$ inhomogeneity is identical between the two acquisitions, as is common for reversed-gradient methods. For each scanning session an additional $\mathrm{B}_{0}$ field map was also obtained for validation. See Table 1 for details of the scanning parameters.

\begin{tabular}{|c|c|c|c|c|c|c|c|c|c|c|c|c|c|c|c|c|c|c|c|c|c|}
\hline sequence & TBW & $\mathrm{D}[\mathrm{ms}]$ & Gz & Gy & $\mathrm{TH}$ [mm] & RES [mm] & TR [ms] & $\mathrm{TE}(\mathrm{s})[\mathrm{ms}]$ & $\mathrm{FA}[\mathrm{o}]$ & ESP [ms] & $\mathrm{R}$ & TA & SL & $\mathrm{FOV}[\mathrm{mm}]$ & $\mathrm{ss} 02$ & $\mathrm{ss03}$ & ss04 & ss05 & ss06 & sg02 & sg03 \\
\hline EPI GRE & 2.6 & $6 / 8 / 10$ & pos/neg & ap & 1.0 & 1.5 & 5940 & 20 & 75 & 0.66 & 4 & 1:05 & 128 & $192 \times 192$ & $\checkmark$ & $\checkmark$ & $\checkmark$ & $\checkmark$ & $\checkmark$ & $\checkmark$ & $\checkmark$ \\
\hline EPI GRE & $5.2 / 7.2$ & 10 & pos/neg & ap & 1.0 & 1.5 & 5940 & 20 & 75 & 0.66 & 4 & 1:05 & 128 & $192 \times 192$ & & & & $\checkmark$ & $\checkmark$ & $\checkmark$ & $\checkmark$ \\
\hline EPI SE & 2.6 & $6 / 8 / 10$ & pos/neg & ap & 1.0 & 1.5 & 15970 & 50 & 90 & 0.66 & 4 & $2: 56$ & 128 & $192 \times 192$ & $\checkmark$ & $\checkmark$ & $\checkmark$ & $\checkmark$ & $\checkmark$ & & \\
\hline EPISE & 2.6 & 10 & pos/neg & $a p / p a$ & 1.0 & 2.0 & 15970 & 50 & 90 & 0.66 & 2 & 2:08 & 128 & $192 \times 192$ & $\checkmark$ & $\checkmark$ & $\checkmark$ & $\checkmark$ & $\checkmark$ & & \\
\hline BO map & -- & -- & -- & ap & 2.0 & 2.0 & 570 & $3.0 / 4.02$ & 46 & -- & -- & 1:52 & 128 & $192 \times 192$ & $\checkmark$ & $\checkmark$ & $\checkmark$ & $\checkmark$ & $\checkmark$ & $\checkmark$ & $\checkmark$ \\
\hline
\end{tabular}

Table 1. Summary of the EPI protocol parameters: TBW = time-bandwidth product in the slice-encoding direction, $D=$ duration of a slice-encoding pulse, Gz = polarities of slice encoding, Gy = directions of phase-encoding gradient (where 'ap' stands for anterior-to-posterior and 'pa' stands for posterior-to-anterior), $\mathrm{TH}=$ slice thickness, RES $=$ nominal in-plane voxel size, $T R=$ repetition time, $T E=$ echo time(s), $F A=$ flip angle, ESP = nominal EPI echo spacing, $R=$ in-plane acceleration factor, $\mathrm{TA}=$ time of acquisition, $\mathrm{SL}=$ number of slices, $\mathrm{FOV}=$ field of view. The right section of the table marks which data were acquired for each of the subjects. and slice bandwidths. The spin-echo EPI may be advantageous over gradient-echo EPI at 7T because of 120 the potential for through-slice dephasing in the gradient-echo, which could cause signal loss exactly in those regions that suffer the most from distortion.

123 scans were acquired consecutively. Both the SE and GE EPI data were acquired near-axially (see above) at $1241.5 \mathrm{~mm}$ in-plane resolution and a slice thickness of $1 \mathrm{~mm}$ using TBW=2.6. To demonstrate the effect of 125 slice bandwidth on the slice distortion, we acquired multiple runs with RF pulse durations $D=6,8$ and 10 126 ms (which corresponds to BW=433, 325 and $260 \mathrm{~Hz}$, respectively), and for a subset of four subjects the 127 GE data with TBW=5.2 and 7.2 and $D=10 \mathrm{~ms}$ (BW=520 and $720 \mathrm{~Hz}$, respectively).

128 To test whether the overeasy method could be used to simultaneously estimate slice-encoding 129 distortion and phase-encoding distortion, for a subset of 5 subjects we also acquired SE EPI data where 
we alternated both the slice-select gradient polarity and the phase-encoding gradient polarity. These data were acquired with $2 \mathrm{~mm}$ in-plane resolution and 1- $\mathrm{mm}$ slice thickness using TBP=2.6 and $D=10 \mathrm{~ms}$, with alternating phase-encoding directions: anterior-to-posterior and posterior-to-anterior (four datasets in total).

To allow us to directly compare the distortion-correction results across all acquired slice-encoding bandwidths and between the SE and GE EPI data, for each subject all datasets acquired with positive sliceencoding gradients were co-registered together and then all datasets acquired with negative sliceencoding gradients were co-registered together using rigid (i.e., 6 degrees of freedom) registration calculated using the FLIRT tool of the FSL software package (Jenkinson et al., 2002; Jenkinson and Smith, 2001). The voxel shift deformation fields were calculated for each reversed slice-encoding volume pair using the topup reversed-gradient method as implemented in FSL (Andersson et al., 2003; Smith et al., 2004) from SE and GE data separately. While topup is mainly intended for correcting distortion along the phase-encoding direction, it is possible to use this software to correct distortion in any encoding direction, therefore no modifications to the software were needed. The topup software performs simultaneous motion correction of the input dataset to correct for potential distortion-independent shifts between volumes acquired with reversed slice-encoding gradient polarities; this effectively provides a coregistration across positive and negative slice-encoding gradient polarity volume pairs, ignoring any potential motion in the distorted direction. Two distortion correction approaches were evaluated and applied to GE EPI data: (1) using the voxel shifts in the slice direction estimated from the SE EPI overeasy pairs, consistent with the typical use case of the topup method, and (2) using voxel shifts in the slice direction estimated from the GE EPI data themselves, to test whether any signal loss present due to through-slice dephasing would bias the results.

Slice-distortion can also be estimated using a conventional $\mathrm{B}_{0}$ field map, provided that the sliceencoding bandwidth and gradient direction are known, and this distortion estimate should be equivalent to that given by overeasy. Therefore, to validate the overeasy-based correction, voxel shift maps based on separately acquired $\mathrm{B}_{0}$ field map scans resampled to the EPI space were calculated, then distorted along the EPI phase-encoding direction so that they more accurately matched the distorted EPI data. The resulting voxel shift maps were estimated for different TBW and $D$ combinations and compared pairwise with those estimated from the corresponding overeasy data at a voxel-by-voxel basis, and the consistency between these two estimates was evaluated by calculating the correlation coefficient between the $\mathrm{B}_{0^{-}}$ fieldmap-based and the overeasy-based estimates. 
Finally, a two-step topup approach was used to correct both slice-encoding and phase-encoding

162 distortion an additional SE EPI dataset acquired with two pairs of reversed gradient directions-i.e., the

163 SE EPI data were acquired four times with both positive and negative phase-encoding and slice-encoding

164 directions. For correction, the phase-encoding distortion correction was performed first using the two

165 pairs of data, after which the slice-encoding distortion correction was performed.

\section{Results}

167 We found clear geometric distortion to occur in the slice-encoding direction in all GE and SE EPI datasets 168 acquired with the proposed overeasy method which allows for reversing slice-select gradient polarity, as

169 illustrated in Figure 1. Example pairs of images with alternating slice-encoding directions are shown in

170 Figure 2, where distortions up to several slice-thicknesses are observable in regions of strong $B_{0}$ field

171 inhomogeneity. The regions near the frontal sinus where distortion is the most severe were magnified to

172 visualize the magnitude of voxel shifts; to aid in the visual inspection of these displacements, a horizontal

173 red line-placed in the same location in scanner coordinates across the two images of the overeasy pairs-

174 is included as a reference. This simple reversal of the slice-encoding gradient polarities added into the

175 pulse sequences provides a concrete demonstration of the slice-distortion effect. We then corrected the

176 slice-select direction distortion in the overeasy pairs of images by applying topup method, where either

177 SE or GE EPI reference data of opposite slice-encoding gradient polarities were used to derive a 178 deformation field (Figure 3).

179 Figure 2 presents representative example sagittal reformats of the original distorted GE EPI data 180 acquired with TBW=2.6 and $D=10 \mathrm{~ms}(\mathrm{BW}=260 \mathrm{~Hz})$ with positive and negative gradient polarities (marked 181 with red arrows at the images) for three different subjects, together with the corrected data based on 182 either the deformation estimated from the SE EPI data or from the GE EPI data themselves. An overview 183 of the distorted and corresponding corrected data for all remaining subjects is presented in 184 Supplementary Figure 1. 


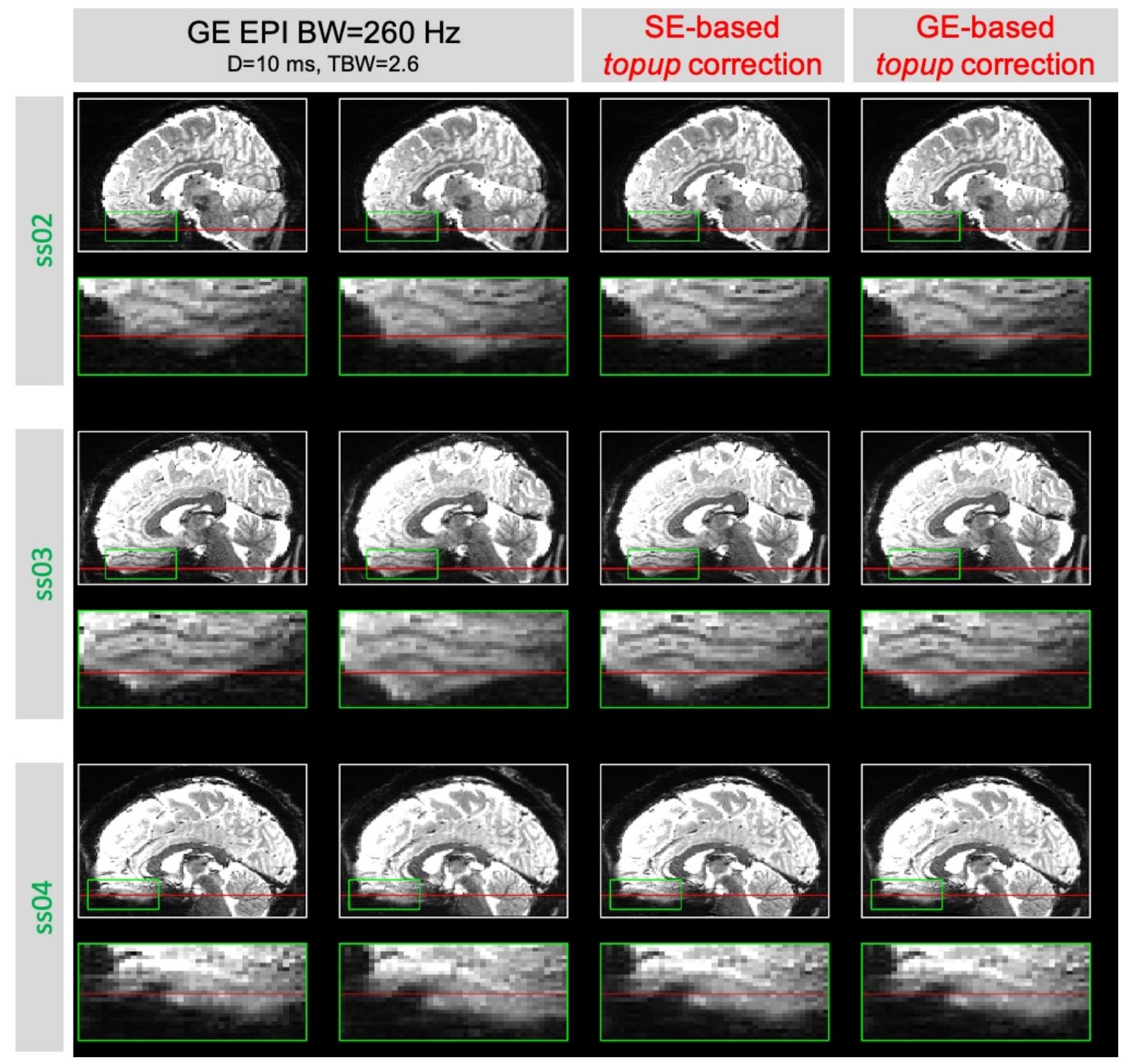

Figure 2. Example sagittal reformats of GE EPI datasets from three different subjects (ss02, ss03 and ss04), acquired with 1$\mathrm{mm}$ slice thickness, 1.5- $\mathrm{mm}$ in-plane resolution, positive and negative slice-encoding gradient direction, slice-encoding pulse duration $D=10 \mathrm{~ms}$ and TBW=2.6, which corresponds to $B W=260 \mathrm{~Hz}$ (left). The positive $\left(+\mathrm{G}_{z}\right)$ and negative $\left(-\mathrm{G}_{\mathrm{z}}\right)$ slice encoding directions are indicated by red arrows. Corresponding slices distortion-corrected using SE-based (middle) and GE-based (right) data. The green box represents the regions shown in the magnified inserts, which show distortion in the slice encoding direction in the frontal sinus region, and the red horizontal line is positioned the same location in all images as a reference to help visualize the amount of displacement in the image data. The corresponding images for all remaining subjects and BW values tested are presented in Supplementary Figure 1. 
As expected, the extent of distortion in the slice-encoding direction was the most pronounced in

186

187

188

189

190

191

192

193

194

195

196

197

198

199

200

201

202

203

204

205

206

207

208

209

210 the images acquired with the lowest slice-encoding gradient bandwidth of $260 \mathrm{~Hz}$, which corresponded to TBW of 2.6 and $D$ of $10 \mathrm{~ms}$, and gradually decreased with increasing bandwidth values. Figure 3 presents a comparison of the example sagittal reformats from GE and SE EPI datasets acquired for one subject with different BW values ranging from 260 to $720 \mathrm{~Hz}$, and both positive and negative slice-encoding gradient polarity. A summary of the examples for all the datasets and the corresponding distortion corrected slices from all remaining subjects can be found in Supplementary Figure 2. Nearly identical results were obtained from our SE and GE EPI data as shown in Figure 4 which summarized the correlations between the voxel shift maps resulting from both topup approaches and the voxel shift map derived from the field map scan. The correlation values are similar for SE- and GE-topup, suggesting that SE EPI data may not be needed when using GE data with such thin slices, even at 7T.

All voxel shift maps generated using topup and calculated from the separately acquired $\mathrm{B}_{0}$ field map scans demonstrated similar spatial distribution of the shift values, reaching $\pm 2 \mathrm{~mm}$ which corresponds to two times the slice thickness, as can be seen in Figure 4A for a representative subject. The correlation coefficients calculated between topup and field map-based maps within the brain mask were highest for the lowest BW values (the most distorted datasets) reaching 0.58-0.82 (Figures $4 A \& B$ ) and the correlation coefficient decreased with the increasing BW. The lower correlation coefficient seen for the data with the lowest level of distortion is likely due to the smaller displacements estimated in the presence of noise (i.e., lower "displacement-to-noise ratio"). The mean values of absolute voxel shifts calculated within the brain were also similar for both approaches, with topup estimations being slightly higher overall and SE-topup estimations being slightly higher than GE-topup. (Figure 4C). Images acquired with lower slice-gradient BW demonstrated larger mean absolute voxel shift values (Figure 4C) over larger spatial area of the brain (Figure 4A) with the decreasing trend for increasing BWs, as expected.

We also demonstrated a two-step approach using topup to correct distortion in phase-encoding and slice-select direction applied to four SE EPI volumes acquired separately with $A \rightarrow P$ and $P \rightarrow A$ phaseencoding gradient direction, and positive and negative slice-encoding gradient direction (Figure 5). 

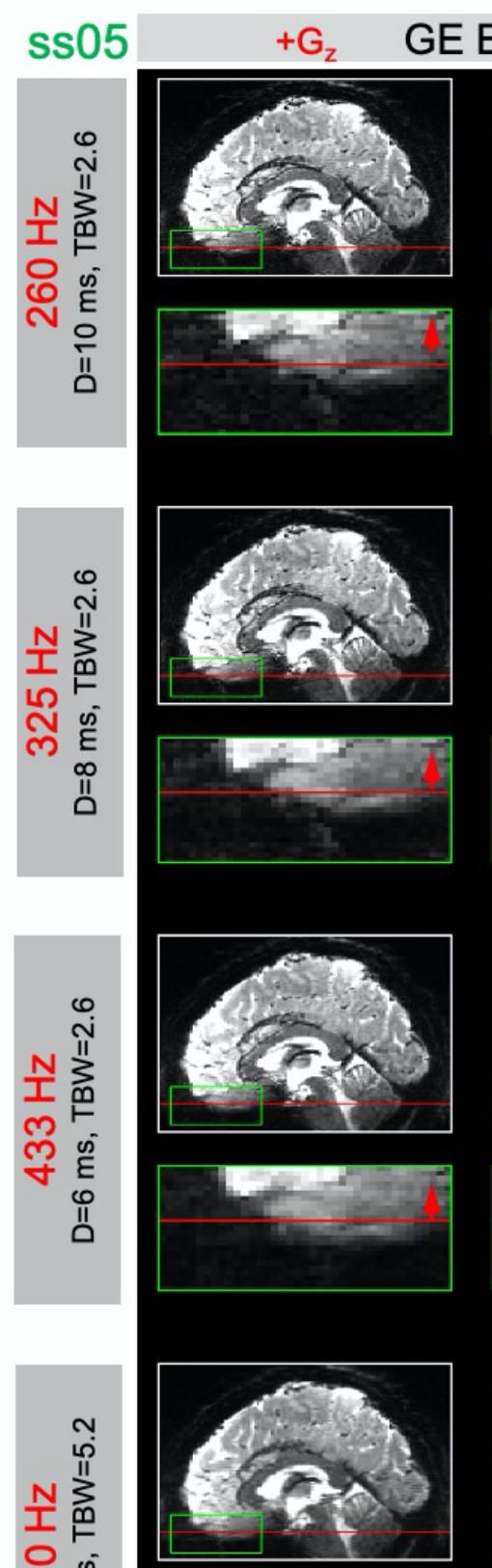

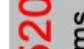

$$
\text { i }
$$
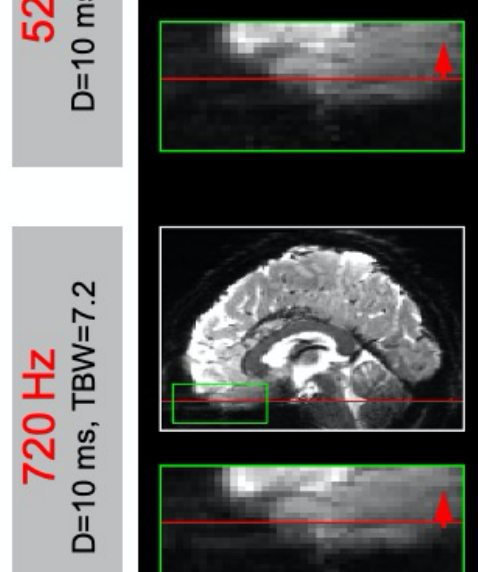
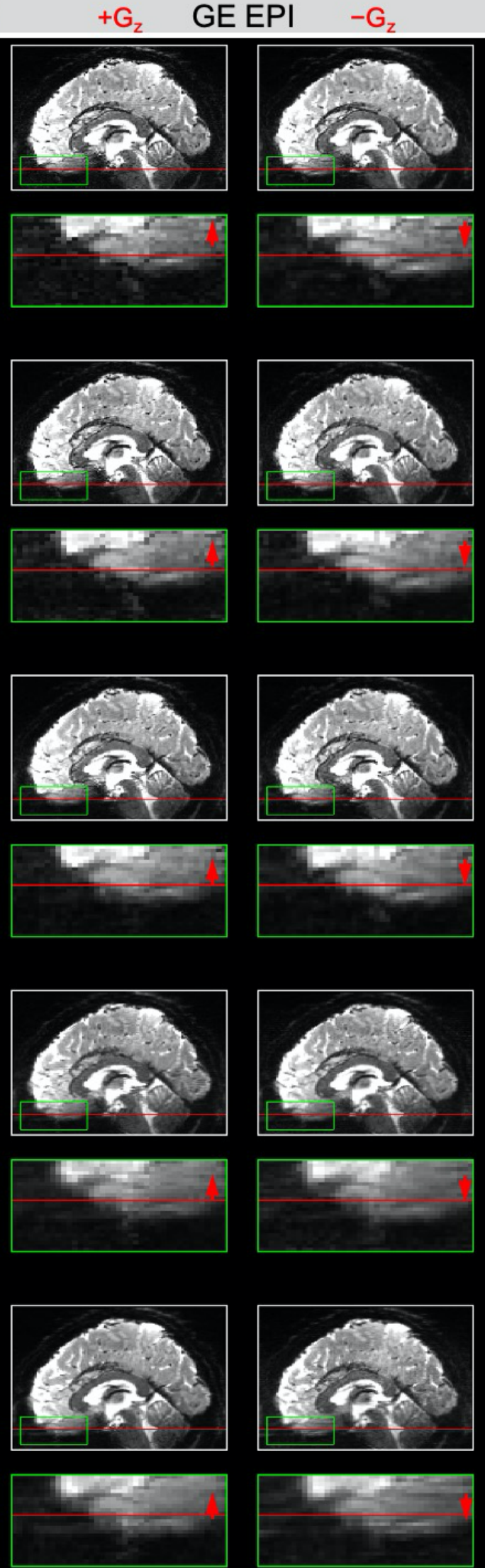
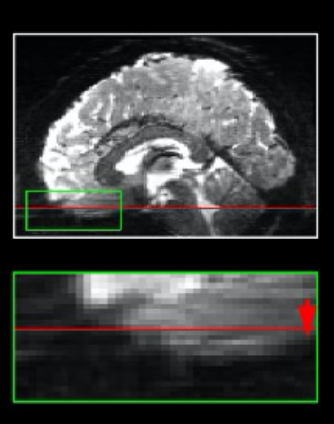

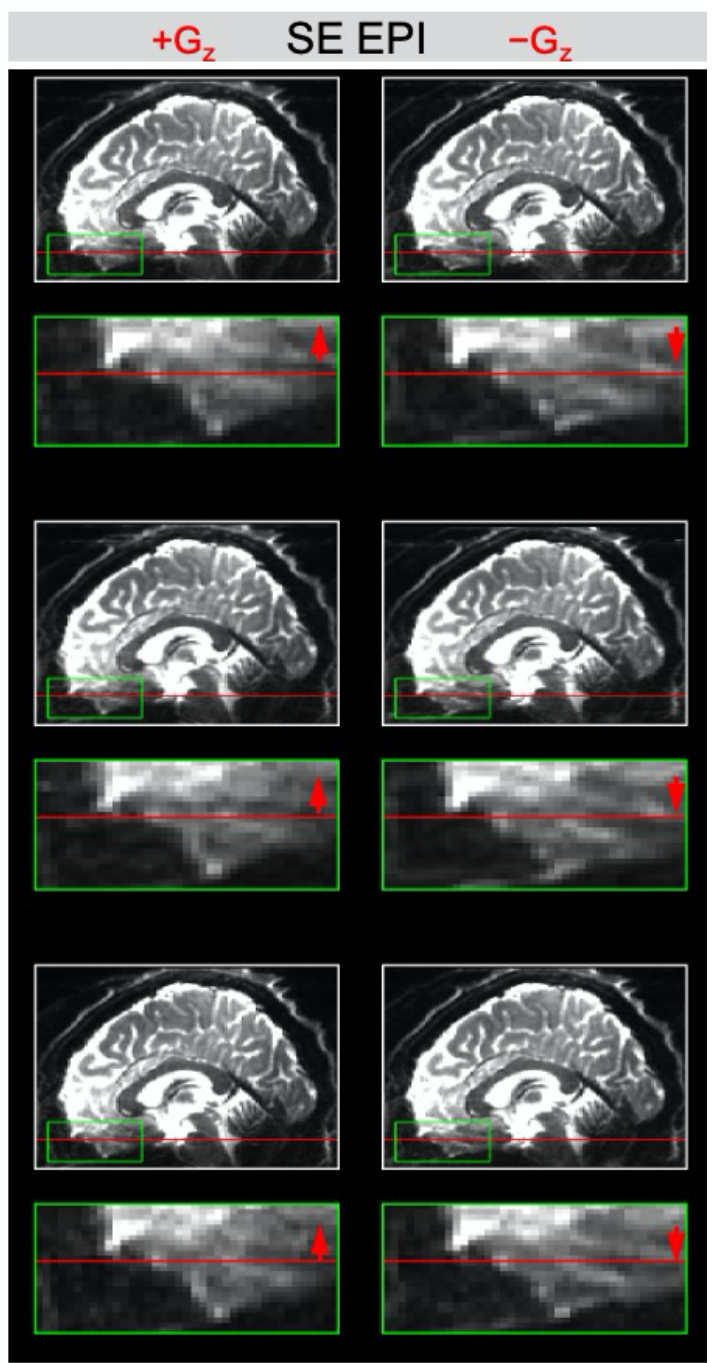

Figure 3. Example sagittal reformats of gradient-echo (GE, left) and spin-echo (SE, right) EPI datasets from a representative subject (ss05), with 1-mm slice thickness and $1.5-\mathrm{mm}$ in-plane resolution. Positive $\left(+G_{z}\right.$ left $)$ and negative $\left(-G_{z}\right.$, right) slice-encoding direction (indicated by red arrows). The rows are ordered according to increasing slice-encoding BW values achieved by changing either the slice-select/slice-refocus pulse duration $(D)$ or timebandwidth product (TBW); see text for details. The horizontal red lines are included in the same position in each pair of images to help visualize shift of voxels due to distortion; magnified inserts correspond to a region indicated by the green box where distortion is pronounced. A summary of the data acquired for all remaining subjects is presented in Supplementary Figure 2. 


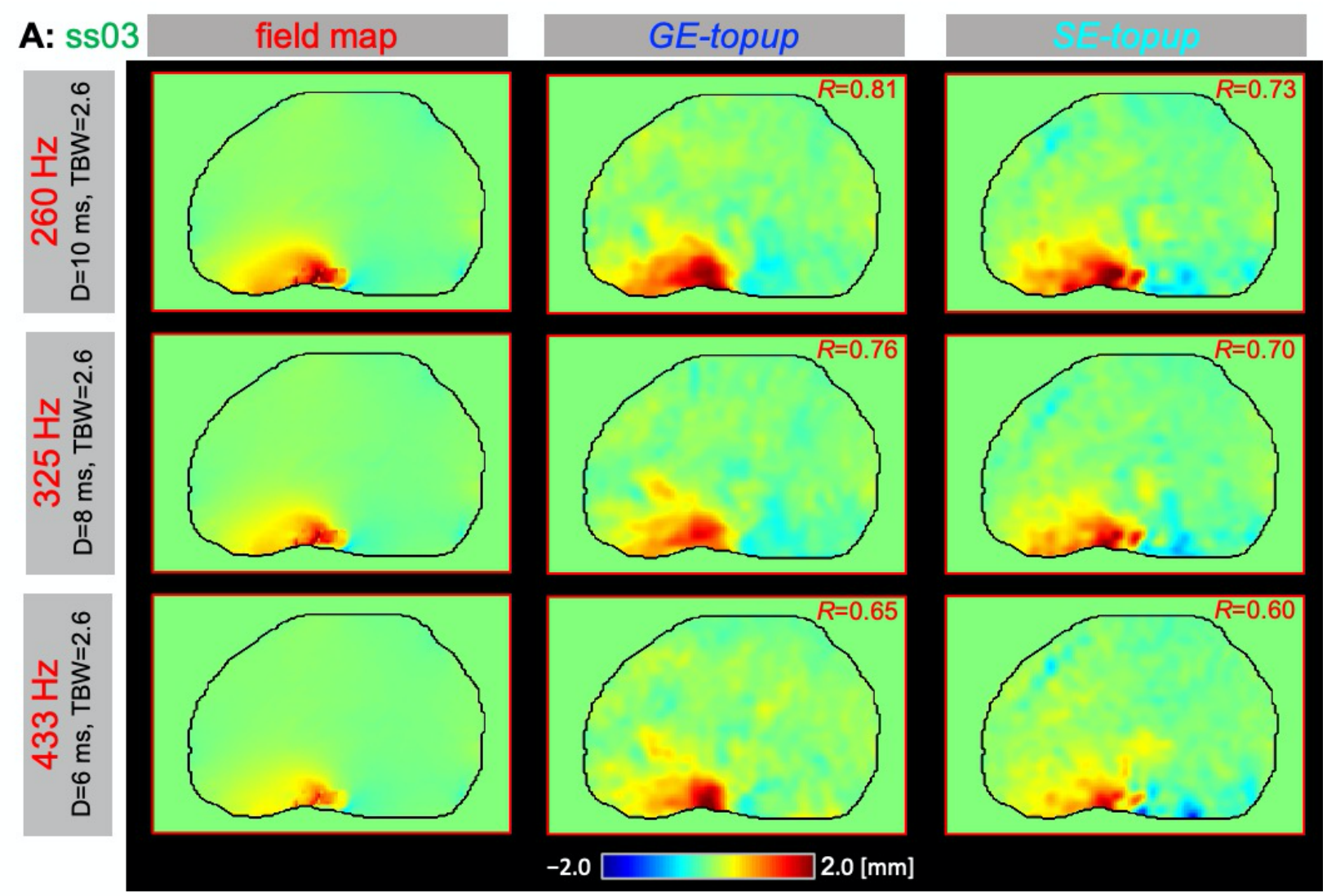

\begin{tabular}{|c|c|c|c|c|c|c|c|c|}
\hline B: tc & & & & & & & & \\
\hline & $G E-t o$ & & & & & $S E$-tor & & \\
\hline $\mathrm{BW}[\mathrm{Hz}]$ & 260 & 325 & 433 & 520 & 720 & 260 & 325 & 433 \\
\hline ss02 & 0.82 & 0.80 & 0.70 & & & 0.77 & 0.58 & 0.66 \\
\hline ss03 & 0.81 & 0.76 & 0.65 & & & 0.73 & 0.70 & 0.60 \\
\hline ss04 & 0.80 & 0.59 & 0.77 & & & 0.79 & 0.74 & 0.75 \\
\hline ss 05 & 0.68 & 0.62 & 0.72 & 0.50 & 0.32 & 0.67 & 0.57 & 0.60 \\
\hline ss06 & 0.76 & 0.79 & 0.74 & 0.62 & 0.51 & 0.80 & 0.75 & 0.76 \\
\hline sg02 & 0.65 & 0.66 & 0.64 & 0.45 & 0.35 & & & \\
\hline sg03 & 0.73 & 0.67 & 0.71 & 0.68 & 0.52 & & & \\
\hline
\end{tabular}

\section{C: mean of absolute voxel shift, all subjects}

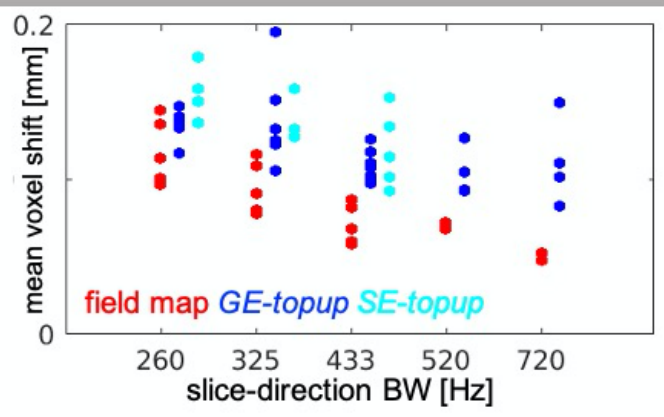

Figure 4. (A) Example sagittal reformats of voxel shift maps in the slice-encoding direction calculated for a representative subject (ss03) using the reverse-gradient-based (i.e., topup) (left) and conventional $\mathrm{B}_{0}$ field map-based approach (middle) accompanied by the correlation coefficient calculated for each pair within the brain region (right). (B) Summary of the correlation coefficients calculated for pairs of the voxel shift maps across all subjects and BW values. (C) Comparison of topup and field map-based mean absolute voxel shift values for all subjects and BWs, with the blue points representing the voxel shift estimated from topup and the cyan points representing the corresponding voxel shift estimated from the $B_{0}$ field map. 


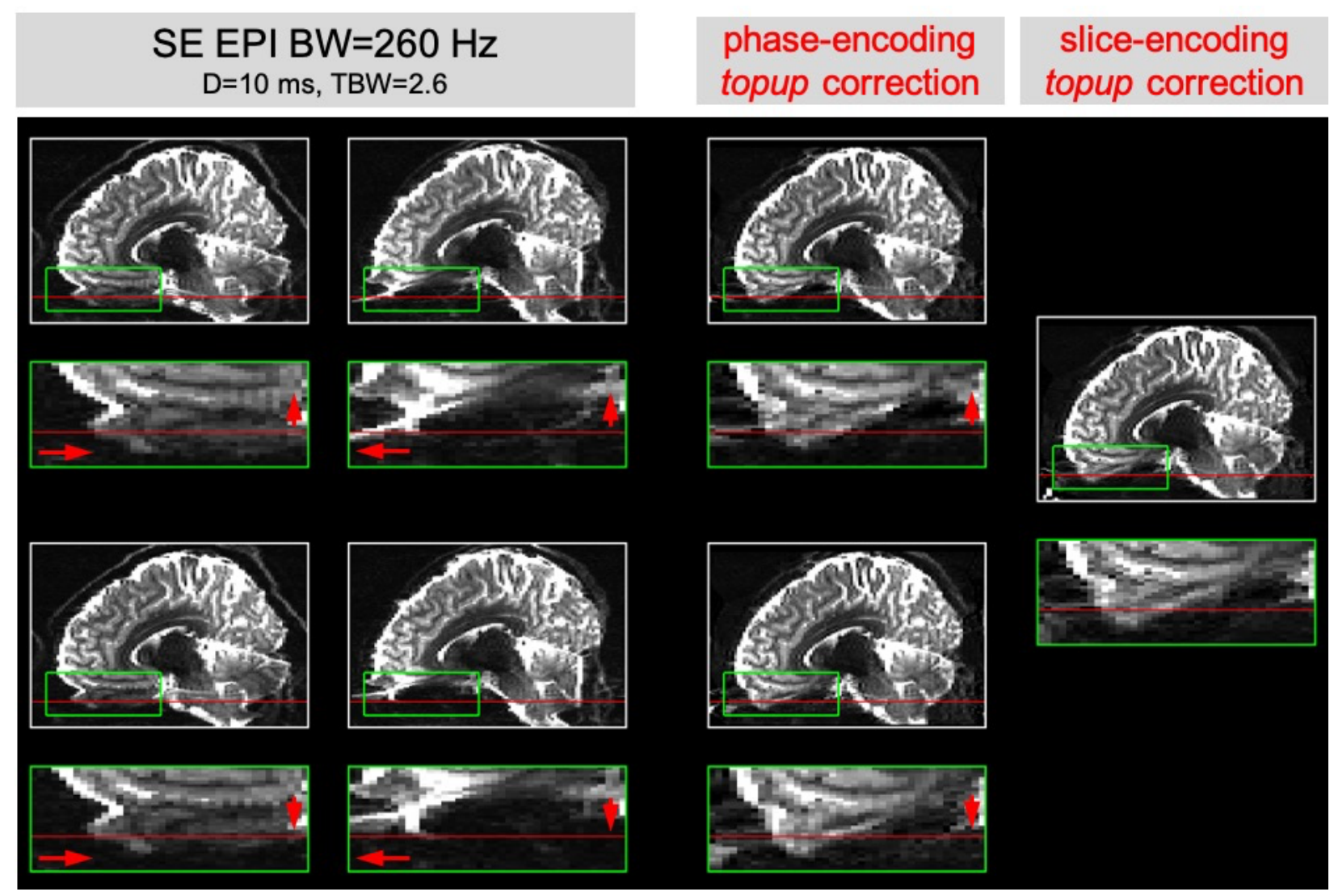

Figure 5. Example sagittal reformats of the SE EPI dataset, acquired axially with 1-mm slice thickness, $2.0 \mathrm{~mm}$ in-plane resolution, $\mathrm{A} \rightarrow \mathrm{P}$ and $\mathrm{P} \rightarrow \mathrm{A}$ phase-encoding gradient direction (first and second column, respectively), positive and negative slice-encoding gradient direction (top and bottom row, respectively), slice-encoding pulse duration $D=10 \mathrm{~ms}$ and TBW=2.6, which corresponds to $B W=260 \mathrm{~Hz}$; positive $\left(+G_{z}\right)$ and negative $\left(-G_{z}\right)$ slice-encoding direction, as well as $A \rightarrow P$ and $P \rightarrow A$ phaseencoding direction are indicated by red arrows; corresponding data distortion-corrected using topup method in phaseencoding direction (third column) and slice-encoding direction (fourth column); magnified inserts show distortion in the frontal sinus region with red horizontal line positioned in the same location in all panels.

\section{Discussion}

212 In this work we presented a simple method for quantifying distortion in the slice-select direction, and 213 demonstrated that modern high-resolution (i.e., thin-sliced) 7T fMRI data contain geometric distortion in 214 the slice direction, with a voxel shifts that may exceed the slice thickness. We were able to measure these 215 distortions from either SE or GE-based acquisitions, indicating that for these thin-slice protocols through216 slice dephasing does not prevent accurate estimations from GE-based data, although whether this finding 217 generalizes to other GE-based protocols will depend on several factors including slice thickness, TE, slice 218 prescription, brain region, and $B_{0}$ shim quality. While these slice-encoding distortions are smaller in 219 magnitude than the well-known phase-encoding distortions, for accurate alignment of data across 
different MRI data, such as alignment of 2D EPI data to 3D anatomical reference data, slice distortion must be addressed to ensure accuracy of the alignment.

While slice distortion can be readily estimated using conventional $\mathrm{B}_{0}$ field map methods, provided the slice-select bandwidth and gradient direction are known, here we used a simple approach to demonstrate in a concrete way the level of slice-distortion that does not require any knowledge of the RF pulses. Our method, based on pairs of EPI data, is faster than conventional methods that acquire an explicit $B_{0}$ field map, which generally require multiple minutes, making them less practical-especially in cases where estimating distortion multiple times during a session is desirable, which may be the case in subjects prone to motion. Because magnetic-susceptibility-induced $B_{0}$ inhomogeneity changes with head position, and, in particular, with head orientation relative to the $B_{0}$ axis, updating the $B_{0}$ inhomogeneity and the associated image distortion periodically during the imaging session can help ensure consistent accuracy of the correction throughout the session. EPI-based field maps are another possibility and have some advantages over $B_{0}$ field maps acquired with conventional encoding (Hutton et al., 2002), provided that the distortions are not too severe. These have also been extended to estimating dynamic changes in distortion during fMRI runs (Lamberton et al., 2007). Still, an advantage of acquiring pairs of reversegradient images is that it provides a means to validate distortion correction based on conventional $\mathrm{B}_{0}$ field maps: if the $B_{0}$ field map yields an accurate estimate of the image distortion, applying the $B_{0}$-fieldmapbased correction to each image of the reversed-gradient pair should result in two identical corrected images. Also, it is possible to estimate slice-encoding distortion present in conventional (i.e., non-EPI) image encoding methods as well using the overeasy method, time permitting, if the slice-select RF pulse bandwidth can be set to be identical to that of the target acquisition.

Another important advantage of reverse-gradient methods for estimating slice-encoding distortion is that they can also help remove distortions due to eddy currents. Since the slice-select gradient amplitude can be larger than other gradient blips, it can be a source of eddy currents that induce $B_{0}$ inhomogeneity in the image and therefore distortion (Varadarajan et al., 2021). Reversing the sliceencoding gradient will reverse the eddy currents and therefore both eddy current and susceptibility distortions can be corrected. This is challenging to achieve with conventional $\mathrm{B}_{0}$ field maps, unless again the $B_{0}$ field map uses the same slice-select gradient as the target acquisition so that both the $B_{0}$ field map and the target acquisition experience the same eddy currents from the slice-select gradient, which would allow the $B_{0}$ field map to measure the same $B_{0}$ inhomogeneity (due to both eddy currents and susceptibility gradients) that is present during the target acquisition. 
When $\mathrm{B}_{0}$ inhomogeneity becomes severe, the distortions along the phase-encoding direction can grow sufficiently large as to induce "singularities" in the EPI data where the compressive distortion results in what is known as "voxel pile-up" artifacts. These artifacts cannot be corrected when using a single phase-encoding polarity, but they can be resolved when reversed-gradient pairs are available. In principle it is possible for "slice pile-up" to occur, although given that the slice-encoding bandwidth is considerably higher (20x and more) than typical phase-encoding bandwidths this is unlikely. In more moderate cases, $\mathrm{B}_{0}$ inhomogeneity will cause a spatially-varying voxel size in the phase-encoding direction, due to the geometric expansion and compression of the image (Wang et al., 2021). While $\mathrm{B}_{0}$ inhomogeneity is often viewed as causing mainly a shift in the slice position, if the $\mathrm{B}_{0}$ field changes within the slice along the slice direction it can similarly cause an expansion or compression. However, given that relatively low sliceencoding bandwidths are mainly used to achieve thin slices, and the $\mathrm{B}_{0}$ field is generally smoothly varying in space, it is also unlikely that the $B_{0}$ field will change considerably at the spatial scale of these thin slices. Future work will consider the effects of $B_{0}$ inhomogeneity on the true resolution of the image in the slice direction.

A final practical consideration is on how to apply this distortion correction to the data. While here the image pairs were corrected directly, using a nonlinear warping followed by an interpolation, in practice, especially in high-resolution applications, it is preferable to mathematically compose all transformations of the data to minimize the number of interpolation steps (Glasser et al., 2013; Polimeni et al., 2018). Because the distortion in the slice-encoding direction will typically be far smaller than that of the phase-encoding direction, most of the displacements in the slice-encoding direction will likely be sub-voxel shifts, which will induce blurring when interpolation is performed. Higher-order interpolation, combined with image grid upsampling, can also help mitigate resolution losses due to interpolation.

Here we focused on demonstrating the distortion in the slice-encoding direction by reversing the gradient polarity of the slice-select gradient, and demonstrated that the bandwidths used in modern fMRI acquisitions do lead to perhaps unexpectedly large levels of distortion the slice direction. This approach

277 we demonstrated above (see Figure 5). While here we acquired four images, but reversing each gradient direction, in principle only one pair is needed, e.g., a positive-slice-encode and positive-phase-encode image followed by a negative-slice-encode and negative-phase-encode image, to save time. In this case, the differential distortion between the two images is no longer constrained to be only along the sliceencoding direction or along the phase-encoding direction, rather it would be at an angle partway between 282 these two directions, where this angle would depend on the ratio of the bandwidths. For example, if the 
283 phase-encoding direction was in $y$, the slice-encoding in $z$, and the phase-encoding bandwidth were 5

284 times larger than the slice-encoding bandwidth, the differential distortion between the two images of the pair would be along a line about $\tan ^{-1}(1 / 5)=11^{\circ}$ off of the $y$ axis.

\section{Conclusions}

287 Distortion in the slice-encoding direction up to several millimeters was detected in data acquired at 7T 288 using a standard high-resolution fMRI protocol. Reducing bandwidth in the slice-encoding direction allows 289 for acquiring thin slices but introduces vulnerability to geometric distortion due to $B_{0}$ inhomogeneity. 290 While this distortion is lower than that found in the phase-encoding direction, it is large enough to reduce 291 accuracy in the alignment of data acquired using different protocols. Both GE and SE EPI acquisitions were 292 used to acquire reversed-gradient data by alternating the polarity of the slice-encoding gradient along 293 with the RF pulse frequency offset. These pairs of data allowed distortion correction using a conventional 294 reverse-gradient method, implemented in topup. This slice-encoding gradient reversal can also be used 295 as a means to validate geometric distortion correction based on acquired $B_{0}$ field maps. The proposed 296 reversed-gradient-based distortion correction methods, termed overeasy, is beneficial in that it reduces 297 the scan time needed to estimate distortion, just as existing EPI-based reversed-gradient methods do for phase-encoding distortion correction, and helps ensure that the relevant distortions from both susceptibility effects and eddy currents are estimated properly.

\section{Acknowledgements}

301 Special thanks to Ned Ohringer and Kyle Droppa for help with subject recruitment and to Azma Mareyam 302 and Dr. Jason Stockman for hardware support. This research has been supported by NIH NIBIB (P41303 EB015896, P41-EB030006 and R01-EB019437), by the BRAIN Initiative (NIMH R01-MH111419 and K99-

$304 \mathrm{MH} 120054)$, and by the Athinoula A. Martinos Center for Biomedical Imaging, and made possible by NIH Shared Instrumentation Grants S10-RR023401, S10-RR023043 and S10-RR020948. 


\section{References}

Andersson, J.L.R., Skare, S., Ashburner, J., 2003. How to correct susceptibility distortions in spin-echo echo-planar images: application to diffusion tensor imaging. Neuroimage 20, 870-88. https://doi.org/10.1016/S1053-8119(03)00336-7

Blazejewska, A.I., Witzel, T., Wald, L.L., Polimeni, J.R., 2017. Correction of EPI geometric distortion in slice direction using reversed slice-select gradients and topup. Proc. Int. Soc. Magn. Reson. Med. 25, 1650.

Bowtell, R.W., McIntyre, D.J.O., Commandre, M.-J., Glover, P.M., Mansfield, P., 1994. Correction of Geometric Distortion in Echo Planar Images, in: ISMRM. p. 441.

Chang, H., Fitzpatrick, J.M., 1992. A Technique for Accurate Magnetic Resonance Imaging in the Presence of Field Inhomogeneities. IEEE Trans. Med. Imaging 11, 319-329. https://doi.org/10.1109/42.158935

de Zwart, J.A., Van Gelderen, P., Kellman, P., Duyn, J.H., 2002. Application of sensitivity-encoded echoplanar imaging for blood oxygen level-dependent functional brain imaging. Magn. Reson. Med. 48, 1011-1020. https://doi.org/10.1002/mrm.10303

Glasser, M.F., Sotiropoulos, S.N., Wilson, J.A., Coalson, T.S., Fischl, B., Andersson, J.L., Xu, J., Jbabdi, S., Webster, M., Polimeni, J.R., Van Essen, D.C., Jenkinson, M., 2013. The minimal preprocessing pipelines for the Human Connectome Project. Neuroimage 80, 105-124. https://doi.org/10.1016/j.neuroimage.2013.04.127

Griswold, M.A., Jakob, P.M., Chen, Q., Goldfarb, J.W., Manning, W.J., Edelman, R.R., Sodickson, D.K., 1999. Resolution enhancement in single-shot imaging using simultaneous acquisition of spatial harmonics (SMASH). Magn. Reson. Med. 41, 1236-1245. https://doi.org/10.1002/(SICI)15222594(199906)41:6<1236::AID-MRM21>3.0.CO;2-T

Hargreaves, B.A., Worters, P.W., Pauly, K.B., Pauly, J.M., Koch, K.M., Gold, G.E., 2011. Metal-induced artifacts in MRI. Am. J. Roentgenol. 197, 547-555. https://doi.org/10.2214/AJR.11.7364

Holland, D., Kuperman, J.M., Dale, A.M., 2010. Efficient Correction of Inhomogeneous Static Magnetic Field- Induced Distortion in Echo Planar Imaging. 2Neurolmage 50, 1-18. https://doi.org/10.1016/j.neuroimage.2009.11.044.Efficient

Hopper, T.A.J., Vasilić, B., Pope, J.M., Jones, C.E., Epstein, C.L., Song, H.K., Wehrli, F.W., 2006. Experimental and computational analyses of the effects of slice distortion from a metallic sphere in an MRI phantom. Magn. Reson. Imaging 24, 1077-1085. https://doi.org/10.1016/j.mri.2006.04.019

Hutton, C., Bork, A., Josephs, O., Deichmann, R., Ashburner, J., Turner, R., 2002. Image distortion correction in fMRI: A quantitative evaluation. Neuroimage 16, 217-40. 


\section{https://doi.org/10.1006/nimg.2001.1054}

Jenkinson, M., Bannister, P., Brady, M., Smith, S., 2002. Improved optimization for the robust and accurate linear registration and motion correction of brain images. Neuroimage 17, 825-41.

Jenkinson, M., Smith, S., 2001. A global optimisation method for robust affine registration of brain images. Med. Image Anal. 5, 143-156.

Jezzard and Balaban, 1995. Correction for Geometric Distortion in Echo Planar Images from B0 Field Variations. Magn. Reson. Med. 34, 65-73.

Jezzard, P., 2012. Correction of geometric distortion in fMRI data. Neuroimage 62, 648-51. https://doi.org/10.1016/j.neuroimage.2011.09.010

Keil, B., Triantafyllou, C., Hamm, M., Wald, L.L., 2010. Design optimization of a 32-channel head coil at 7T, in: Intl Soc Mag Reson Med. p. 1493.

Lamberton, F., Delcroix, N., Grenier, D., Mazoyer, B., Joliot, M., 2007. A new EPI-based dynamic field mapping method: application to retrospective geometrical distortion corrections. JMRI 26, 747-55. https://doi.org/10.1002/jmri.21039

Lu, W., Pauly, K.B., Gold, G.E., Pauly, J.M., Hargreaves, B.A., 2009. SEMAC: Slice Encoding for Metal Artifact Correction in MRI. Magn. Reson. Med. 62, 66-76. https://doi.org/10.1002/mrm.21967.SEMAC

Morgan, P.S., Bowtell, R.W., McIntyre, D.J.O., Worthington, B.S., 2004. Correction of spatial distortion in EPI due to inhomogeneous static magnetic fields using the reversed gradient method. JMRI 19, 499507. https://doi.org/10.1002/jmri.20032

Polimeni, J.R., Renvall, V., Zaretskaya, N., Fischl, B., 2018. Analysis strategies for high-resolution UHF-fMRI data. Neuroimage 168, 296-320. https://doi.org/10.1016/j.neuroimage.2017.04.053

Smith, S.M., Jenkinson, M., Woolrich, M.W., Beckmann, C., Behrens, T.E.J., Johansen-Berg, H., Bannister, P.R., De Luca, M., Drobnjak, I., Flitney, D.E., Niazy, R.K., Saunders, J., Vickers, J., Zhang, Y., De Stefano, N., Brady, J.M., Matthews, P.M., 2004. Advances in functional and structural MR image analysis and implementation as FSL. Neuroimage 23 Suppl 1, S208-19.

Studholme, C., Constable, R.T., Duncan, J.S., 2000. Accurate alignment of functional EPI data to anatomical MRI using a physics-based distortion model. IEEE Trans. Med. Imaging 19, 1115-27. https://doi.org/10.1109/42.896788

Sumanaweera, T.S., Glover, G.H., Sumanaweera, T.O., Adler, J.R., 1993. MR susceptibility misregistration correction. IEEE Trans. Med. Imaging 12, 251-259. https://doi.org/10.1109/42.232253

Varadarajan, D., Balasubramanian, M., Park, D.J., Witzel, T., Stockmann, J.P., Polimeni, J.R., 2021. Characterizing the acquisition protocol dependencies of BO field mapping and the effects of eddy 
bioRxiv preprint doi: https://doi.org/10.1101/2021.12.16.472821; this version posted December 19, 2021. The copyright holder for this preprint (which was not certified by peer review) is the author/funder. All rights reserved. No reuse allowed without permission. currents and spoiling. Proc. Int. Soc. Magn. Reson. Med. 29, 3552.

371 Wang, J., Nasr, S., Roe, A.W., Polimeni, J.R., 2021. Evaluation of spatial blur induced by preprocessing and distortion in UHF fMRI data. Proc Intl Soc Mag Reson Med 29, 3126. 